Erschienen in: Deutsch als Fremdsprache 45.4. Zeitschrift zur Theorie und Praxis des Deutschunterrichts für Ausländer. - München/Berlin: Langenscheidt, 2008. S. 208-215.

Annette Klosa

\title{
Zur Optimierung von Wortbildungsangaben in DaF-Wörter- büchern auf der Basis systematischer Korpusuntersuchungen
}

\section{Wortbildung in DaF-Wörterbüchern}

Angaben zur Wortbildung nehmen in Lernerwörterbüchern keinen zentralen, aber auch keinen zu vernachlässigenden Raum ein. So sind zahlreiche Veröffentlichungen zum Thema erschienen, ${ }^{1}$ die sich zumeist darin einig sind, dass ,ein Wörterbuch [...], das sich besonders als Lernerwörterbuch versteht, [...] auch die sprachlichen Einheiten als Stichwörter aufnehmen [sollte], die in so hohem Maße am Ausbau des Wortschatzes beteiligt sind wie die verschiedenen Wortbildungselemente" (Poethe 1996: 189).

Neben der Aufnahme von Wortbildungselementen als Lemmata sind für (Lerner-)Wörterbücher weitere Möglichkeiten entwickelt worden, Angaben zur Wortbildung zu machen, und zwar insbesondere die Integration von Angaben zu Ableitungen aus dem Stichwort oder zu Zusammensetzungen mit dem Stichwort innerhalb eines Wortartikels. Gerade für Lerner des Deutschen als Fremdsprache ist anzunehmen, dass umfangreiche Angaben dazu, in welche anderen Wortbildungsprodukte ein Stichwort eingeht, die Bedeutungsangaben eines Stichwortes be- reichern, differenzieren und außerdem signalisieren, dass die verzeichneten Wortbildungsprodukte geläufig sind und , also zum zentralen Wortschatz" (Barz 1995: 18) gehören. Daneben können sie das Hervorbringen eigener korrekter Wortbildungsprodukte ermöglichen, indem größere Mengen regelhafter Bildungen die Aufmerksamkeit auf die sprachlichen Regularien lenken, bewusstes Lernen ermöglichen und so den Spracherwerb unterstützen (vgl. Fandrych/Tschirner 2007: 196ff.).

Schließlich können (eher undurchsichtige) Komposita neben den entsprechenden Simplizia in die Stichwortliste integriert werden,

\footnotetext{
${ }^{1} \mathrm{Vgl}$. zur Wortbildung im allgemeinen einsprachigen Wörterbuch insbesondere die Beiträge von Allhoff (1992), Barz (2001), Bergenholtz (2000), Holly (1986), Motsch (1982), Mugdan (1984), Muller (1989) und Rettig (1989). Zu Wortbildungsangaben in Lernerwörterbuchern vgl. vor allem Barz $(1995 ; 2002)$, van der Colff (1998), Kempcke (1992), Poethe (1996) und Seelig (2001). Untersuchungen zur Wortbildung in zweisprachigen Wörterbüchern werden hier nicht ber ucksichtigt.
} 
wobei hier (wie auch beim Erfassen von Wortbildungsprodukten zu einem Stichwort) aufgrund des begrenzten Druckraumes eine Auswahl aus allen möglichen Wortbildungsprodukten getroffen werden muss. Es ist deshalb bei der Wörterbuchkonzeption immer zu klären, ,inwieweit durch die Angabe von Wortbildungselementen und Wortbildungsregeln [...] das Wörterbuch entlastet oder dem Benutzer eine Hilfestellung bei der Analyse und Synthese nicht aufgenommener Wortbildungen gegeben werden kann" (Mugdan 1984: 238).

In den meisten einsprachigen Wörterbüchern des Deutschen und auch in den Lernerwörterbüchern wird die Gebildetheit des Stichwortes selbst nicht systematisch analysiert und beschrieben, ' obwohl allgemein angenommen wird, dass „Wortbildung einen Beitrag zur Durchsichtigkeit und Erschließbarkeit des Wortschatzes leisten" kann (Holly 1986: 204). Vor dem Hintergrund der allgemeinen Platznot im gedruckten Wörterbuch scheint es tatsächlich aber für Lernerwörterbücher wichtiger zu sein, Wortbildungsmittel und Wortbildungsprodukte zu verzeichnen. $^{2}$

Neben den metalexikografischen Vorschlägen zur Behandlung von Wortbildung im Wörterbuch bieten die (Lerner-)Wörterbuicher des Deutschen konkrete Umsetzungen. Wortbildung im (Lerner-)Wörterbuch findet sich an verschiedenen Stellen der Makro- und Mikrostruktur und dient auch dazu, die Vernetztheit des Wortschatzes aufzuzeigen. Ein paar Beispiele sollen dies illustrieren. ${ }^{3}$

\footnotetext{
${ }^{1}$ Holly (1986: 204) weist darauf hin, dass „der Wortbildungsaspekt meist von etymologischen Ideen überlagert oder auf eine rein morphologische Auffassung reduziert worden" ist, und Motsch (vgl. 1982: 69) bemängelt, dass solche Angaben nur gelegentlich gemacht werden.

2 Anders kann z. B. ein Online-Wörterbuch, das nicht der Platzbeschränkung der gedruckten Seite unterliegt, vorgehen; vgl. hierzu z. B. www.elexiko.de, ein Wortschatzinformationssystem zum Deutschen, das am Mannheimer Institut fur Deutsche Sprache erarbeitet wird. Näheres hierzu in Klosa (2005).

Als Beispielwörterbücher werden hier ausschlieBlich die drei Großwörterbücher für Deutsch als Fremdsprache herangezogen, obwohl sich sicherlich auch der Blick in die inzwischen erschienenen Kompakt- und Taschenwörterbücher lohnen würde, z. B. LTWDaF, HWDaF, PKWDaF.
}

Der Wortartikel Land in WaGWDaF verzeichnet einige Komposita mit Land als Grund- oder Bestimmungswort:

Land 1. abgegrenztes Stück Erdboden, Grundstück; Zus Ackerland, Grünland, Waldland, Weideland. 2. Festland. 3. Wald, Wiese u. Feld (im Gegensatz zum städtischen Lebensraum); Zus Landbevölkerung, Landleben, Landluft. [...]

Daneben sind folgende Komposita mit Land als Bestimmungswort (hier nur eine Auswahl aus den Nomen) als eigenes Stichwort aufgeführt: Landbesitz, Landenge, Länderspiel, Landesbank, Landkarte, Landkreis, Landtier, Landweg, Landwirt. Hinzu kommen Derivate wie landen oder ländlich.

LGWDaF unterscheidet in den Wortartikeln nach Komposita mit dem Stichwort als Bestimmungswort (notiert als ,|| K-") oder als Grundwort (notiert als „॥-K“), z. B.:

Landschaft: 1. ein Teil der Oberfläche der Erde || K-: Landschafts-, -pflege, -schutz || -K: Berg-, Gebirgs-, Hügel-, Küsten-, Sumpf-, Winter-. [...]

Zugleich wird vermittelt, ob ein Fugenelement eingeschoben wird. Außerdem werden Derivate explizit eingeführt, z. B.:

Landwirt: j-d, der selbstständig auf e-m Bauernhof arbeitet $u$. ihn leitet II hierzu Landwirtin.

WDaF verzeichnet innerhalb der Angaben zur „Wortfamilie“ im Wortartikel Land auch Derivate, die an alphabetisch entfernter Stelle im Wörterbuch erscheinen würden, z. B. Gelände, Ausland. Dieses Wörterbuch macht schließlich Angaben dazu, zu welchem Kernwort einer Wortfamilie ein Stichwort zählt bzw. gebildet wurde, z. B. ländlich und Landschaft zu Land. Außerdem finden sich hier (wie auch in den anderen genannten Wörterbüchern) Wortbildungselemente als Stichwörter, z. B.:

2-los /bildet mit einem Subst. als erstem Be-
standteil Adjektive; drückt aus, dass das im ers-
ten Bestandteil Genannte nicht vorhanden ist/:
$\uparrow$ z. B. kraftlos, niveaulos. (Lerner-)Wörterbücher enthalten also Angaben zur Wortbildungsproduktivität eines Lexems, die besonders für Wörterbuchtypen mit textbezogener Funktion (vgl. Bergenholtz 2000: 773), d. h. für solche, die bei der Rezeption, Produktion und Übersetzung eines Textes konsultiert werden, relevant sind. In einer 
Textproduktionssituation ist $\mathbf{z}$. B. fraglich, ob ein Kompositum mit oder ohne Fugenelement gebildet wird. Einem Wortartikel wie dem oben gezeigten Landschaft ist dies zu entnehmen. Beim Verfassen von Texten kann außerdem der Wunsch entstehen, beim Ausdruck durch die Wahl eines Hyponyms zu variieren. Dafür sind Angaben zu möglichen Komposita hilfreich, wie sie die oben gezeigten Wortartikel anbieten. Umgekehrt sollen unbekannte Komposita in einer Textrezeptionssituation in ihrer Bedeutung erschlossen werden. Es ist auch hierfür nützlich, wenn möglichst viele Komposita entweder selbst als Stichwort behandelt oder in anderen Wortartikeln erfasst sind. Außerdem kann das Textverständnis durch das Erlernen von Wortfamilien unterstützt werden, weshalb der explizite Hinweis auf Zusammenhänge innerhalb einer Wortfamilie wie in WDaF sinnvoll erscheint. Schließlich können Angaben zur Wortbildungsproduktivität ermöglichen, Wortbildungsmuster $\mathrm{zu}$ erlernen, die $\mathrm{zu}$ eigenen korrekten Bildungen und Verwendungen sowie regelhaften Ad-hoc-Bildungen befähigen.

\section{Kritische Überprüfung anhand von Korpusdaten}

Nach diesem Einblick in die derzeitige Praxis der DaF-Wörterbücher soll das Vorgehen nun kritisch überprüft werden. Dabei stehen drei Angabearten im Zentrum:

1. Erfassung von Komposita in der Stichwortliste,

2. Erfassung von Wortbildungsprodukten $\mathrm{zu}$ einem Stichwort,

3. Erfassung von Wortbildungselementen.

\subsection{Erfassung von Komposita in der Stichwortliste}

Gängige Praxis von Lernerwörterbüchern ist es, in die Stichwortliste Simplizia und nichtdurchsichtige Ableitungen und Zusammensetzungen aufzunehmen, wobei undurchsichtige Komposita solche sind, „bei denen die Bedeutung des zusammengesetzten Ausdrucks nicht bloß aus den individuellen Kompositionsgliedern und dem Wortbildungsmuster abzuleiten ist" (van der Colff 1998: 194). Durchsichtige Komposita werden dagegen, wie oben gezeigt, am Ende eines Wortartikels verzeichnet. Die folgende Übersicht zur Wörterbuchstrecke Land - Landzunge aus den drei untersuchten DaF-Großwörterbüchern (s. Tab. 1,S.211) zeigt, wie unterschiedlich die Lemmatisierung von nominalen Komposita gehandhabt wird. Zusätzlich ist verzeichnet, welche dieser Bildungen zu den 4.000 häufigsten Lexemen im Häufigkeitswörterbuch von Jones/Tschirner (2006) zählen und welche zu den 30.000 Stichwörtern der korpusbasierten Wortgrundformenliste DEREWo ${ }^{1}$ des IDS Mannheim gehören.

Nur zwei der Komposita (Landkreis, Landwirtschaft) zählen zu den 4.000 häufigsten Wörtern bei Jones/Tschirner (2006); diese sind immerhin in allen untersuchten Wörterbüchern erfasst. 27 Komposita aus DEREWO sind in den Wörterbüchern lemmatisiert: elf Stück nur in einem der Wörterbücher, neun in zwei und nur sieben in allen Wörterbüchern. Hierbei fällt auf, dass WaGWDaF eine ganze Reihe von Komposita, die auch in DEREWo enthalten sind, als einziges Wörterbuch erfasst: Landesbank, -ebene, -gruppe, -regierung, -teil, -vater und Landsitz. Es ist zu vermuten, dass dies deshalb der Fall ist, weil WaGWDaF korpusbasiert erarbeitet wurde (vgl. WaGWDaF: 7). Grundsätzlich ist von den insgesamt 47 in den Wörterbüchern verzeichneten Komposita die Hälfte (nämlich 23) nur in jeweils einem Wörterbuch verzeichnet, etwa ein Drittel (nämlich 16) in zwei Wörterbüchern und nur der Rest (nämlich acht) in allen drei Wörterbüchern.

Dem stehen die 20 lemmatisierten Bildungen gegenüber, die nicht zu den 30.000 häufigsten in DEREWo gehören: Zwölf von ihnen sind in nur einem Wörterbuch als Stichwort erfasst, sechs in zwei und zwei in allen Wörterbüchern. Gerade bei diesen fragt sich, warum sie aufgenommen wurden, andere Bildungen dagegen nicht. DEREWO bietet nämlich noch weitere rund 60 offensichtlich höher frequente Bildungen mit Land als Bestimmungswort, die - berücksichtigt man ausschließlich die Frequenz - eher Aufnahmekandidaten gewe-

\footnotetext{
1 Die DeReWo-Wortliste basiert auf dem „Deutschen Referenzkorpus" DeReKo des IDS Mannheim. Sie wurde „auf der Grundlage herkömmlicher lexikographischer Kriterien mit besonderer Berucksichtigung der Gebrauchshäufgkeit in Form von Korpusfrequenz" zusammengestellt (Dokumentation zur „Korpusbasierten Wortgrundformenliste DeReWo, v-30000g-2007-1231-0.1“: 2).

2 Mit Asterisk sind solche Komposita in Tab. 1 (s. S. 211) markiert. die das WDaF nur als Eintrag im sogenannten "Kompositumsblock" verzeichnet. Das WDaF klassifiziert diese Bildungen offensichtlich als durchsichtig.
} 


\begin{tabular}{|c|c|c|c|c|}
\hline LGWDaF & WDaF & WaGWDaF & Jones/Tschirner & DeReWo \\
\hline & & Landbesitz & & \\
\hline Landeanflug & & & & Landeanflug \\
\hline Landebahn & Landebahn & & & Landebahn \\
\hline Landenge & Landenge & \begin{tabular}{|l|} 
Landenge \\
\end{tabular} & & \\
\hline Landeplatz & & & & Landeplatz \\
\hline \multirow[t]{5}{*}{ Länderspiel } & & Länderspiel & & Länderspiel \\
\hline & & Landesbank & & Landesbank \\
\hline & & Landesebene & & Landesebene \\
\hline & & Landesgruppe & & Landesgruppe \\
\hline & & Landeshauptfrau & & \\
\hline Landeshauptmann & & \begin{tabular}{|l|} 
Landeshauptmann \\
\end{tabular} & & Landeshauptmann \\
\hline \multirow[t]{2}{*}{ Landeskunde } & & Landeskunde & & \\
\hline & & \begin{tabular}{|l|} 
Landesmeister \\
\end{tabular} & & Landesmeister \\
\hline \multirow[t]{6}{*}{ Landesrat } & & & & Landesrat \\
\hline & & Landesrecht & & \\
\hline & & Landesregierung & Landesregierung & Landesregierung \\
\hline & & Landesteil & & Landesteil(e) \\
\hline & & Landesvater & & Landesvater \\
\hline & Landesverrat & \begin{tabular}{|l|} 
Landesverrat \\
\end{tabular} & & \\
\hline \multirow[t]{2}{*}{ Landflucht } & & Landflucht & & \\
\hline & $\begin{array}{l}\text { Landfriedens- } \\
\text { bruch*2 }\end{array}$ & Landfriedensbruch & & Landfriedensbruch \\
\hline Landgericht & & \begin{tabular}{|l|} 
Landgericht \\
\end{tabular} & & Landgericht \\
\hline Landkarte & Landkarte* & Landkarte & & Landkarte \\
\hline Landkreis & Landkreis* & Landkreis & Landkreis & Landkreis \\
\hline \multicolumn{5}{|l|}{ Landmann } \\
\hline Landplage & & Landplage & & \\
\hline Landrat & & \begin{tabular}{|l|} 
Landrat \\
\end{tabular} & & Landrat \\
\hline Landratsamt & & Landratsamt & & Landratsamt \\
\hline \multicolumn{5}{|l|}{ Landratte } \\
\hline \multicolumn{5}{|l|}{ Landregen } \\
\hline & & Landschulheim & & \\
\hline & & \begin{tabular}{|l|} 
Landsitz \\
\end{tabular} & & \begin{tabular}{|l} 
Landsitz \\
\end{tabular} \\
\hline Landsmann & Landsmann & \begin{tabular}{|l|} 
Landsmann \\
\end{tabular} & & \begin{tabular}{|l|} 
Landsmann \\
\end{tabular} \\
\hline Landstraße & & Landstraße & & Landstraße \\
\hline \multirow[t]{2}{*}{ Landstreicher } & & Landstreicher & & \\
\hline & Landstreitkräfte* & Landstreitkräfte & & \\
\hline Landstrich & Landstrich* & \begin{tabular}{|l} 
Landstrich \\
\end{tabular} & & Landstrich \\
\hline \multirow[t]{2}{*}{ Landtag } & Landtag* & $\begin{array}{l}\text { Landtag } \\
\end{array}$ & & Landtag \\
\hline & & Landtier & & \\
\hline \multicolumn{5}{|l|}{ Landungsbrücke } \\
\hline \multicolumn{5}{|l|}{ Landungssteg } \\
\hline \multicolumn{5}{|l|}{ Landurlaub } \\
\hline Landweg & & Landweg & & Landweg \\
\hline \multicolumn{5}{|l|}{ Landwein } \\
\hline Landwirt & & Landwirt & & Landwirt \\
\hline Landwirtschaft & Landwirtschaft* & \begin{tabular}{|l|} 
Landwirtschaft \\
\end{tabular} & Landwirtschaft & Landwirtschaft \\
\hline Landzunge & Landzunge* & Landzunge & & \\
\hline
\end{tabular}

Tab. 1: Wörterbuchstrecke Land - Landzunge 


\begin{tabular}{|c|c|c|c|c|}
\hline LGWDaF & WDaF & WaGWDaF & Jones/Tschirner & DeReWo \\
\hline \multirow[t]{5}{*}{ Ackerland } & & Ackerland & & Ackerland \\
\hline & Bundesland & Bundesland & & Bundesland \\
\hline & & Festland & & Festland \\
\hline & & Grünland & & Grünland \\
\hline & Industrieland & & & Industrieland \\
\hline \multicolumn{5}{|l|}{ Landarzt } \\
\hline Landbevölkerung & & Landbevölkerung & & Landbevölkerung \\
\hline \multicolumn{5}{|l|}{ Landgewinnung } \\
\hline \multicolumn{5}{|l|}{ Ländername } \\
\hline & & Landesamt & & Landesamt \\
\hline Landesgrenze & & & & Landesgrenze \\
\hline \multicolumn{5}{|l|}{ Landeshauptstadt } \\
\hline & & Landesinnere(s) & & Landesinnere(s) \\
\hline & & & & $\begin{array}{l}\text { Landeskranken- } \\
\text { haus }\end{array}$ \\
\hline & & Landeskriminalamt & & \\
\hline \multirow[t]{2}{*}{ Landesparlament } & & & & Landesparlament \\
\hline & & Landesparteitag & & Landesparteitag \\
\hline Landespolitik & & Landespolitik & & Landespolitik \\
\hline Landesregierung & & & Landesregierung & Landesregierung \\
\hline \multirow[t]{3}{*}{ Landessprache } & & Landessprache & & Landessprache \\
\hline & & Landesteil & & \\
\hline & & Landesverband & & Landesverband \\
\hline Landeswährung & & Landeswährung & & Landeswährung \\
\hline \multicolumn{5}{|l|}{ Landeswappen } \\
\hline \multicolumn{5}{|l|}{ Landklima } \\
\hline Landleben & & Landleben & & \\
\hline Landluft & & Landluft & & \\
\hline \multicolumn{5}{|l|}{ Landmasse } \\
\hline \multicolumn{5}{|l|}{ Landpfarrer } \\
\hline \multicolumn{5}{|l|}{ Mittelmeerland } \\
\hline \multirow[t]{3}{*}{ Urlaubsland } & & & & Urlaubsland \\
\hline & Vaterland & & & Vaterland \\
\hline & & Waldland & & \\
\hline Weideland & Weideland & Weideland & & \\
\hline
\end{tabular}

Tab. 2: Komposita innerhalb des Wortartikels Land

sen wären. Frequenz als Auswahlkriterium wird allerdings bei keinem der untersuchten Wörterbücher als zentrales Kriterium genannt; gerade für die Lemmatisierung von Komposita soll ja die Durchsichtigkeit bzw. Undurchsichtigkeit entscheiden. Dass die Anwendung dieses Kriteriums zu sehr unterschiedlichen Ergebnissen führt, zeigt Tab. 1 deutlich. Eine Überprüfung, bestenfalls auch Entschlackung bzw. Ergänzung der Stichwortlisten aufgrund korpusstatistischer Auswertungen besonders im Bereich der Wortbildungen scheint angeraten.

\subsection{Erfassung von Wortbildungsprodukten zu einem Stichwort}

Wie oben anhand der Beispiele gezeigt, verzeichnen die DaF-Großwörterbücher Wortbildungsprodukte zu einem Stichwort. Für die Auswahl postuliert etwa WaGWDaF: „Um dem Lernenden zu zeigen, welche Bildungen von Zusammensetzungen möglich oder gebräuchlich sind, werden bei zahlreichen Stichwörtern [...] die wichtigsten $\mathrm{Zu}$ sammensetzungen aufgeführt.“ (WaGWDaF: 11) In Tab. 2 sind alle zum Stichwort Land erfassten Komposita in den drei untersuchten 
Wörterbüchern zusammengefasst und den Häufigkeitsauszählungen in Jones/Tschirner (2006) und DEREWO gegenübergestellt.

Auch hier fällt auf, dass wenig Übereinstimmung dahingehend herrscht, welche $\mathrm{Zu}$ sammensetzungen mit Land erfasst werden; keines der Komposita ist in allen Wörterbüchern verzeichnet, und von den insgesamt 33 eingetragenen Bildungen sind nur 19 auch in DEREWo enthalten, verfügen also über eine hohe Frequenz. Neben den insgesamt zehn in den Wörterbüchern verzeichneten Bildungen mit Land als Grundwort (immerhin sieben davon sind in DEREWo verzeichnet) bietet DEREWo 34 weitere Komposita wie Alpenland, Einwanderungsland oder Nachbarland, die durchaus ebenfalls aufnehmenswert sind.'

Vor dem Hintergrund der Tatsache, dass zurzeit ,in der Fremdsprachendidaktik dem Einfluss von Häufigkeitseffekten auf den Fremdsprachenerwerb große Beachtung geschenkt" wird (Schmidt 2008: 74), sollte eine korpuslinguistische Sprachbeschreibung nicht nur der ,häufigkeitsorientierten Fremdsprachendidaktik" (vgl. Tschirner 2005) zugrunde liegen, sondern auch den Lernerwörterbüchern. Mit Recht stellt Heine (2008: 3) fest, dass gerade einsprachige Lernerwörterbücher von den Möglichkeiten der Korpuslinguistik „in besonderem Maße profitieren, da die Korpora nicht nur die häufigsten Wörter für die Lemmaselektion, sondern ebenso die häufigsten Strukturen, Wortbildungen, Komposita, ,musterhafte' Beispielsätze und pragmatische Informationen liefern könn(t)en". Dies bestätigen die oben gezeigten exemplarischen Untersuchungen.

\subsection{Erfassung von Wortbildungselementen}

Zur Lemmaselektion gehört auch die Entscheidung, ob und in welchem Umfang Wort-

\footnotetext{
Allerdings ist eine Auswahl unabdingbar; Lemnitzer/ Zinsmeister (2006: 131) bemerken zu Recht: „Große Korpora enthalten viele Belege für die meisten Wortbildungsmuster und durchweg mehr Beispiele, als ein Wörterbuch verzeichnen kann.“

Vgl. hierzu die ausfuhrliche Untersuchung zum WDaF in Barz (2002) und zum LGWDaF in Poethe (1996).

${ }^{3}$ Einzelne verbale Bildungen wie gegenzeichnen oder zwischenlanden sind in LGWDaF dagegen lemmatisiert.

4 Vgl. hierzu den Eintrag Zickenalarm im Neologismenwörterbuch des IDS Mannheim.

Syberspace ist ebenfalls in DeReWo enthalten.
}

bildungselemente lemmatisiert werden sollen. Für die Aufnahme von Präfixen, Suffixen und Affixen in Lernerwörterbücher sind eingangs gute Argumente genannt worden, und auch die drei hier untersuchten Großwörterbücher enthalten (in unterschiedlichem Umfang ${ }^{2}$ ) entsprechende Einträge. Untersucht man näher, welche Wortbildungsmittel erfasst sind, zeigt sich auch hier, dass diese Angaben durch die Auswertung von umfangreichen Korpora präzisiert bzw. ergänzt werden könnten.

LGWDaF verzeichnet $z$. B. die nominalen Präfixe gegen- („sehr produktiv", beispielsweise in Gegenlicht, Gegenbeispiel, Gegenangriff) und zwischen- (,sehr produktiv", beispielsweise in Zwischenbericht, Zwischenlager, Zwischenpause). Es fehlen aber die entsprechenden verbalen Wortbildungsmuster ${ }^{3}$ (z. B. gegenfragen, gegenneigen, gegenlesen, gegenzeichnen bzw. zwischenschieben, zwischenrufen, zwischenspeichern), die verstärkt seit den Neunzigerjahren des 20 . Jahrhunderts belegt sind (vgl. hierzu Klosa 2003; 2004).

Andere, besonders in jüngster Zeit aufgekommene Wortbildungsmuster fehlen noch völlig. So sind im „,Deutschen Referenzkorpus“" des IDS Mannheim über 800 Zusammensetzungen mit Alarm als Grundwort enthalten (natürlich mit jeweils unterschiedlicher Frequenz), z. B. Bergrettungsalarm, Falschalarm, Feueralarm, Hurrikanalarm, aber auch Komposita wie Krötenalarm, Schlampenalarm, Sonnenbrandalarm, Zickenalarm. In solchen Bildungen hat Alarm nicht die ursprüngliche Bedeutung ,Warnsignal vor etwas oder wegen etwas' oder ,Warnsignal für jemanden', sondern signalisiert eher, übertriebene Aufregung wegen etwas oder jemandem ${ }^{6}{ }^{4}$ Zumindest in den großen Lernerwörterbüchern könnte zum Stichwort Alarm diese (vorerst) nur in Komposita realisierte Neubedeutung erwähnt werden.

Ein Beispiel für ein in letzter Zeit zunehmend belegtes Wortbildungselement, das bislang in den untersuchten DaF-Wörterbüchern noch nicht erfasst ist, ist cyber-/Cyber-. Annähernd 200 Bildungen mit cyber- und über 2600

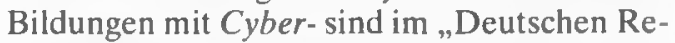
ferenzkorpus" zu finden, unter denen Cyberspace (und seine Zusammensetzungen) natürlich die häufigste ist; immerhin in LGWDaF und WaGWDaF ist Cyberspace lemmatisiert. ${ }^{5}$

Die Auswahl der in einem Lernerwörterbuch zu behandelnden Wortbildungselemente 
sollte im Prinzip genauso korpusgestützt und anhand von Frequenzkriterien erfolgen wie die Auswahl der zu lemmatisierenden Wörter.' Vor diesem Hintergrund ist zu fragen, ob die eben genannten neuen Wortbildungsmittel bzw. -muster tatsächlich in die hier untersuchten DaF-Wörterbücher hätten aufgenommen werden sollen. Poethe (1996: 198) schreibt mit Recht: „Für den kritischen Betrachter von Wörterbüchern [...] wird es immer relativ leicht sein, Wörter oder Wortelemente zu nennen, die er als Lemma im Wörterbuch vermißt. Besonders hinsichtlich der Wortbildungselemente, deren zumindest traditionelles Inventar begrenzt und überschaubar und somit auch recht gut überprüfbar ist, ist das der Fall." Andererseits unterstützt die Darstellung von Wortbildungsmitteln und Wortbildungsmöglichkeiten den Lerner beim rezeptiven Umgang mit Sprache. Besonders in Zeitungstexten treten neben Wortbildungsprodukte mit traditionellen Wortbildungsmitteln neue hinzu. Gerade solche Muster springen ins Auge und machen stutzig, und für ihr Verständnis reicht es nicht, wenn sie gar nicht oder nur anhand einer Bildung im Wörterbuch erklärt werden.

Für die Aufnahme von Wortbildungselementen sollte vermutlich nicht alleine das Frequenzkriterium eine Rolle spielen, sondern ihre Aufnahme ist auch dann zu empfehlen, wenn sie semantisch komplex sind (vgl. das Beispiel Alarm, bei dem eine Neubedeutung hinzukommt) oder wenn das $V_{\text {orwissen }}^{2}$ zu Fehlschlüssen führen würde (vgl. die Beispiele zwischen- und gegen-, bei denen das Vorwis- sen über die nominalen Wortbildungsmuster zu Fehlschlüssen auf die Wortbildungsbedeutung der verbalen Wortbildungsmuster verleiten kann).

\section{Fazit}

Wie Wortbildung in Wörterbüchern behandelt wird und werden kann, ist in der metalexikografischen Forschungsliteratur gut dokumentiert. Von diesen Vorschlägen sind einige in den hier untersuchten großen DaF-Wörterbüchern umgesetzt. Bei der Auswahl der behandelten Stichwörter (Lexeme und Wortbildungselemente) und bei der Erfassung von Wortbildungsprodukten in den Artikeln spielen aber offensichtlich aus großen Textkorpora ermittelte Frequenzangaben eine noch zu geringe Rolle. Dabei ,führt das Prinzip der Korpusgestütztheit zu einem deutlichen Qualitätsgewinn von allgemeinsprachigen Bedeutungswörterbüchern, gerade aber auch von Lernerwörterbüchern“" (Klosa 2007: 119). Dies sollte bei Neuauflagen oder Neuentwicklungen von DaF-Wörterbüchern berücksichtigt werden, damit diese tatsächlich das Ziel des Spracherwerbs unterstützen und zugleich ihrer textbezogenen Funktion gerecht werden können.

\footnotetext{
' $\mathrm{Zu}$ den Chancen, aber auch den Einschränkungen für die automatische Analyse von Korpora im Hinblick auf Wortbildungsmuster vgl. Lemnitzer/Zinsmeister (2006: 133f.).

2 Zur Bedeutung von Vorwissen und semantischer Komplexität als Merkmal des Inputs für den Spracherwerb vgl. Fandrych/Tschirner (2007: 198ff.).
}

\section{Literatur}

Allhoff, Jürgen (1992): Die Berücksichtigung von Affixen in Wörterbüchern. In: G. Meder/A. Dörner (Hg.), Worte, Wörter, Wörterbücher. Lexikographische Beiträge zum Essener linguistischen Kolloquium. Tübingen, 62-86.

Barz, Irmhild (1995): Komposita im Großwörterbuch Deutsch als Fremdsprache. In: I. Pohl/H. Ehrhardt (Hg.), Wort und Wortschatz. Beiträge zur Lexikologie. Tübingen, 13-24.

Barz, Irmhild (2001): Wortbildungsbeziehungen im einsprachigen Bedeutungswörterbuch. In: J. Korhonen (Hg.), 85-100.

Barz, Irmhild (2002): Die Wortbildungsmittel im de Gruyter Wörterbuch Deutsch als Fremdsprache. In: H. E. Wiegand ( $\mathrm{Hg}$.), Perspektiven der pädagogischen Lexikographie des Deutschen II.
Untersuchungen anhand des „de Gruyter Wörterbuchs Deutsch als Fremdsprache". Tübingen, 105-121.

Bergenholtz, Henning (2000): Lexikographie und Wortbildungsforschung. In: I. Barz u. a. (Hg.), Praxis- und Integrationsfelder der Wortbildungsforschung. Heidelberg, 19-30.

van der Colff, Adri (1998): Die Komposita in LANGENSCHEIDTS GROSSWÖRTERBUCH DEUTSCH ALS FREMDSPRACHE. In: H. E. Wiegand ( $\mathrm{Hg}$.), Perspektiven der pädagogischen Lexikographie des Deutschen. Tübingen, 193-207.

Fandrych, Christian / Tschirner, Erwin(2007): Korpuslinguistik und Deutsch als Fremdsprache. Ein Perspektivenwechsel. In: DaF 4, 195-204. 
Hausmann, Franz Josef u. a. (Hg.) (1989): Wörterbücher. Ein internationales Handbuch zur Lexikographie. Erster Teilband. Berlin/New York.

Heine, Antje (2008): Zur Nutzbarkeit der gegenwärtig verfügbaren deutschen Korpora für die Lernerlexikografie Deutsch als Fremdsprache. Anspruch und Wirklichkeit. In: DaF 1, 2-8.

Holly, Werner (1986): Wortbildung und Wörterbuch. In: Lexicographica 2, 195-213.

Kempcke, Günter (1992): Organisationsprinzipien und Informationsangebote in einem Lernerwörterbuch. In: U. Brauße/D. Viehweger (Hg.), Lexikontheorie und Wörterbuch. Wege der Verbindung von lexikologischer Forschung und lexikographischer Praxis. Tübingen, 165243.

Klosa, Annette (2003): gegen-Verben - ein neues Wortbildungsmuster. In: Sprachwissenschaft 28, 467-494.

Klosa, Annette (2004): zwischen-Verben. Ein Zwischenbericht. In: Sprachreport 4, 16-20.

Klosa, Annette (2005): Wortbildung. In: U. Haß (Hg.), Grundfragen der elektronischen Lexikografie. elexiko - das Online-Informationssystem zum deutschen Wortschatz. Berlin/New York, 141-162.

Klosa, Annette (2007): Korpusgestützte Lexikographie: besser, schneller, umfangreicher? In: W. Kallmeyer/G.Zifonun ( Hg.), Sprachkorpora. Datenmengen und Erkenntnisfortschritt. Berlin u. a., 105-122.

Korhonen, Jarmo (Hg.) (2001): Von der mono- zur bilingualen Lexikografie für das Deutsche. Frankfurt a.M. u. a.

Korpusbasierte Wortgrundformenliste DEREWO, v-30000g-2007-12-31-0.1, mit Benutzerdokumentation, http://www.ids-mannheim.de/kl/derewo/,
(C) Institut für Deutsche Sprache, Programmbereich Korpuslinguistik, Mannheim, Deutschland, 2007.

Lemnitzer, Lothar/Zinsmeister, Heike (2006): Korpuslinguistik. Eine Einführung. Tübingen.

Motsch, Wolfgang (1982): Wortbildungen im einsprachigen Wörterbuch. In: E. Agricola u.a. (Hg.), Wortschatzforschung heute. Aktuelle Probleme der Lexikologie und Lexikographie. Leipzig, 62-71.

Mugdan, Joachim (1984): Grammatik im Wörterbuch. Wortbildung. In: H. E. Wiegand (Hg.), Studien zur neuhochdeutschen Lexikographie IV, 237-308 (Germanistische Linguistik 1-3/83).

Müller, Wolfgang (1989): Die Beschreibung von Affixen und Affixoiden im allgemeinen einsprachigen Wörterbuch. In: F. J. Hausmann u. a. (Hg.), 869-882.

Poethe, Hannelore (1996): Wortbildung im Großwörterbuch Deutsch als Fremdsprache. In: I. Barz/M. Schröder (Hg.), Das Lernerwörterbuch Deutsch als Fremdsprache in der Diskussion. Heidelberg, 189-207.

Rettig. Wolfgang (1989): Die Wortbildungszusammenhänge im allgemeinen einsprachigen Wörterbuch. In: F. J. Hausmann u. a. (Hg.), 642649.

Schmidt, Claudia (2008): Grammatik und Korpuslinguistik. Überlegungen zur Unterrichtspraxis DaF. In: DaF 2, 74-80.

Seelig, Barbara (2001): Neue lexikographische Wege der Darstellung in de Gruyters Wörterbuch ,Deutsch als Fremdsprache'. In: J. Korhonen (Hg.), 65-84.

Tschirner, Erwin (2005): Korpora, Häufigkeitslisten, Wortschatzerwerb. In: A. Heine u. a. (Hg.), Deutsch als Fremdsprache. Konturen und Perspektiven eines Faches. München, 133-149.

\section{Wörterbücher/Wortlisten}

DEREWO $=$ Korpusbasierte Wortgrundformenliste DEREWo, v-30000g-2007-12-31-0.1, mit Benutzerdokumentation, http://www.ids-mannheim. de/kl/derewo/, (c) Institut für Deutsche Sprache, Programmbereich Korpuslinguistik, Mannheim, Deutschland, 2007.

elexiko = http://www.elexiko.de (zuletzt eingesehen April 2008).

HWDaF = Hueber Wörterbuch Deutsch als Fremdsprache. Das einsprachige Wörterbuch für Kurse der Grund- und Mittelstufe. Ismaning 2007.

Jones, Randall L./Tschirner, Erwin: Frequency dictionary of German - core vocabulary for learners. London / New York 2006.

LGWDaF = Langenscheidt Großwörterbuch Deutsch als Fremdsprache. Neubearbeitung. $\mathrm{Hg}$. von D. Götz/G. Haensch / H. Wellmann. Berlin u. a. 2008.
LTWDaF = Langenscheidt Taschenwörterbuch Deutsch als Fremdsprache. Neubearbeitung. Hg. von D. Götz/H. Wellmann. Berlin u. a. 2007.

Neologismenwörterbuch = http://www.owid.de/ Neologismen/index.html (zuletzt eingesehen April 2008).

PKWDaF = Pons Kompaktwörterbuch Deutsch als Fremdsprache. Vollständige Neuentwicklung. Stuttgart 2005.

WaGWDaF = Wahrig Großwörterbuch Deutsch als Fremdsprache. Von R. Wahrig-Burfeind. Gütersloh u. a. 2008.

WDaF = Wörterbuch Deutsch als Fremdsprache. Von G. Kempcke unter Mitarbeit von B. Seelig/B. Wolf/E. Tellenbach u.a. Berlin u.a. 2000. 